\title{
Evidence for different origins of the magnetic field effect on current and electroluminescence in organic light- emitting diodes
}

Cite as: Appl. Phys. Lett. 100, 123302 (2012); https://doi.org/10.1063/1.3696051

Submitted: 17 February 2012 . Accepted: 03 March 2012 . Published Online: 20 March 2012

Andreas Buchschuster, Tobias D. Schmidt, and Wolfgang Brütting

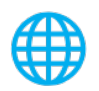

\section{ARTICLES YOU MAY BE INTERESTED IN}

Light extraction and optical loss mechanisms in organic light-emitting diodes: Influence of the emitter quantum efficiency

Journal of Applied Physics 104, 123109 (2008); https://doi.org/10.1063/1.3043800

Enhancement of organic magnetoresistance by electrical conditioning

Applied Physics Letters 92, 193309 (2008); https://doi.org/10.1063/1.2924765

Charge accumulation at organic semiconductor interfaces due to a permanent dipole moment and its orientational order in bilayer devices

Journal of Applied Physics 111, 114508 (2012); https://doi.org/10.1063/1.4724349

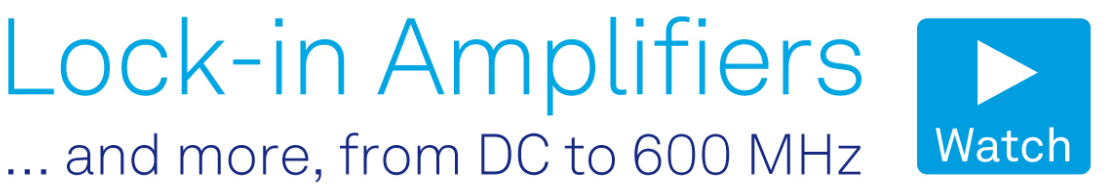




\title{
Evidence for different origins of the magnetic field effect on current and electroluminescence in organic light-emitting diodes
}

\author{
Andreas Buchschuster, Tobias D. Schmidt, and Wolfgang Brütting ${ }^{\text {a) }}$ \\ Institute of Physics, University of Augsburg, 86135 Augsburg, Germany
}

(Received 17 February 2012; accepted 3 March 2012; published online 20 March 2012)

\begin{abstract}
An external magnetic field can change the current through an organic light-emitting diode and the luminance it emits. Existing models predict that both phenomena have the same behaviour and, therefore, a common origin; however, there are indications that they are not completely linked. As a direct proof, we measured the magnetic field effect in multilayer organic light-emitting diodes using $\mathrm{Alq}_{3}$ as emission layer. After successively adding blocking layers, we found a decrease of the magnetic field effect on the current, whereas the effect on the luminance remained at the same level. Thus, both effects can be separated from each other. (C) 2012 American Institute of Physics. [http://dx.doi.org/10.1063/1.3696051]
\end{abstract}

The organic magnetic field effect (OMFE) was discovered in $2003,{ }^{1}$ when it was found that an external magnetic field of a few $10 \mathrm{mT}$ can affect the current and the electroluminescence of an organic light-emitting diode (OLED) based on $\mathrm{Alq}_{3}$ (tris(8-hydroxyquinoline)aluminium). In addition, studies revealed that this effect also occurs in many other organic semiconducting materials based on small molecules or polymers. ${ }^{2-4}$ Until now, the observed changes in current range from about $1 \%$ to $25 \%$, whereas the spread is to a major part, a consequence of the dependence of the effect on the degradation of a device. ${ }^{5}$

In the literature, the magnetic field effect on the luminance (organic magnetoluminance; OML) and the magnetic field effect on the current (organic magnetoresistance; OMR) are said to share a common origin, as both exhibit the same behaviour, such as equal line shapes and full width at half maximum. ${ }^{4,6,7}$ Therefore, the major part of the change in luminance is regarded as being a consequence of the change in current, and so the OML is often neglected. Nonetheless, there are indications that both effects are not as closely related as supposed. ${ }^{8}$

In the present work, we prepared OLEDs with different electron transporting/hole blocking layers and investigated the behaviour of current and electroluminescence in a magnetic field. It was found that the magnitude of the OMR can be significantly reduced, if the injection of holes into the electron transport layer (ETL) is prevented by a blocking layer. Surprisingly, the corresponding OML was not affected by the introduction of blocking layers. This suggests that both effects are spatially separated and, therefore, have to be considered as independent from each other. Furthermore, this approach may offer a handle to design OLEDs that respond to a magnetic field by a change of luminescence without changing the electrical properties.

The devices examined in this article were prepared in a clean-room using glass substrates covered with indium tin oxide (ITO) purchased from Merck. The substrates were structured by photolithography and then cleaned with several solvent steps in an ultrasonic bath for 15 min each. After that

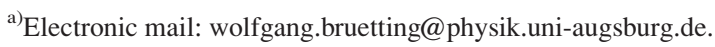

the ITO surface was treated with oxygen plasma and covered with a layer of $30 \mathrm{~nm}$ poly(3,4-ethylenedioxythiophene): poly(styrenesulfonate) (PEDOT:PSS) applied by spincoating. The substrate was then transferred to a glovebox $\left(\mathrm{O}_{2}\right.$ and $\mathrm{H}_{2} \mathrm{O}<5 \mathrm{ppm}$ ) with an attached evaporation-chamber, where the organic and metallic layers were deposited without breaking the vacuum. After fabrication, the devices were placed in a cryostat under high-vacuum conditions, where the I-V-L (current-voltage-luminance) and the magnetic field measurements were performed in. The cryostat was placed between the poles of a Bruker B-E 15v electromagnet. The electrical characterisation was performed with a Keithley 2400 sourcemeter, and the electroluminescence was detected by a non-calibrated silicon photodiode and read out with a Keithley 6514 electrometer. The orientation of the sample surface was parallel to the applied magnetic field.

The value of the OMFE was determined with a method suggested by Desai et al., which reduces the mistake due to drift. ${ }^{9}$ For this purpose, the current before and after the application of the magnetic field was measured in order to calculate the average value of the zero-field current at the time of the actual measurement of the OMFE. The value of the effect is then obtained by the normalised current difference with and without the magnetic field $(\Delta I / I=(I(B)-I(0)) / I(0))$. The effect on the luminance is calculated analogously.

First, a device was fabricated where the PEDOT:PSS is followed by a hole transport layer (HTL) of $70 \mathrm{~nm}$ TPD (N,N'-diphenyl-N, $\mathrm{N}^{\prime}$-bis(3-methylphenyl)-1,1'-biphenyl4,4'-diamine) and a $70 \mathrm{~nm}$ layer of $\mathrm{Alq}_{3}$, which can be used as an electron transport layer and a light-emitting layer (device 1). In addition to its hole transporting properties, TPD also acts as an electron blocking layer. ${ }^{10}$ To provide electrical contact, the organic layers were capped with $20 \mathrm{~nm}$ calcium and $100 \mathrm{~nm}$ aluminium acting as a cathode. The overlap of the structured ITO and the metallic fingers yield pixels with a size of $2 \times 2 \mathrm{~mm}^{2}$.

In order to alter the number of organic layers, in which both electrons and holes are present, the different layers have to be separated by blocking layers. The electrons are already prohibited from reaching the hole transporting TPD layer, thus, in device 1, both charge carriers are able to interact and 


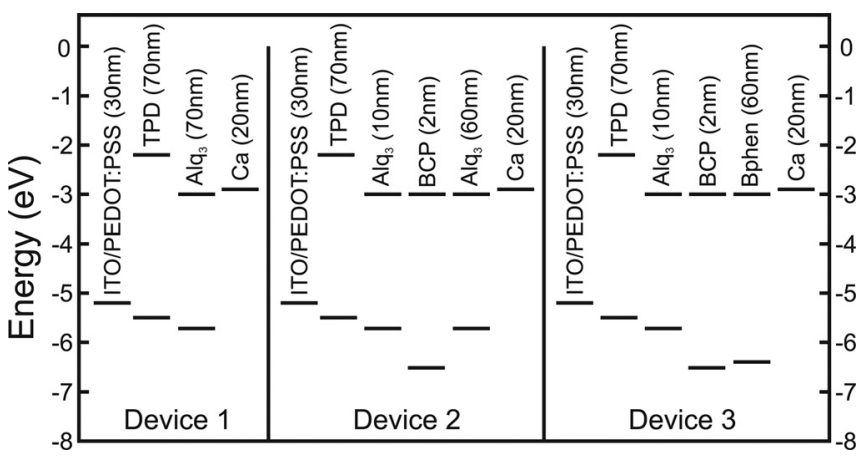

FIG. 1. Schematic HOMO and LUMO levels of the devices under investigation. The values of the energetic levels have been taken from literature as follows: PEDOT:PSS (Ref. 11), TPD (Ref. 12), $\mathrm{Alq}_{3}$ (Ref. 13), BCP (Ref. 14), and BPhen (Ref. 15).

recombine in the $\mathrm{Alq}_{3}$ layer at a path of $70 \mathrm{~nm}$. To suppress the transport of holes into the whole electron transporting regime of the $\mathrm{Alq}_{3}$, a second device was made, in which a thin layer of bathocuproine (BCP) was introduced to divide the $\mathrm{Alq}_{3}$ layer into two regions (device 2). Consequently, the main section where electrons and holes recombine with each other should be an $\mathrm{Alq}_{3}$ layer with a thickness of $10 \mathrm{~nm}$, acting as an emission layer (EML), followed by $2 \mathrm{~nm} \mathrm{BCP \text {, }}$ which acts as a hole blocker ${ }^{16}$ and, therefore, separates the rest of the $\mathrm{Alq}_{3}$, which now acts as an ETL only with a thickness of $60 \mathrm{~nm}$. In a third device, the remaining $60 \mathrm{~nm} \mathrm{Alq}$ ETL was replaced by a layer of BPhen (4,7-diphenyl-1,10phenanthroline) of the same thickness, whereas the rest of the device stayed unchanged (device 3). This material can conduct electrons but the transport of holes is suppressed. ${ }^{15}$ The schematic energy levels of all three devices are illustrated in Fig. 1.

I-V-L measurements have been performed as shown in Fig. 2. By adding the above mentioned hole blocking layers, the current through the different devices is successively reduced and so is the luminance at a given voltage. This could be a consequence of the space charge limited current, ${ }^{17}$ which is dominant in that type of device. Therefore, the total current is reduced when less holes in the ETL are able to compensate the electron space charge at the cathode. Device 3 displays a smaller leakage current than the other two, but

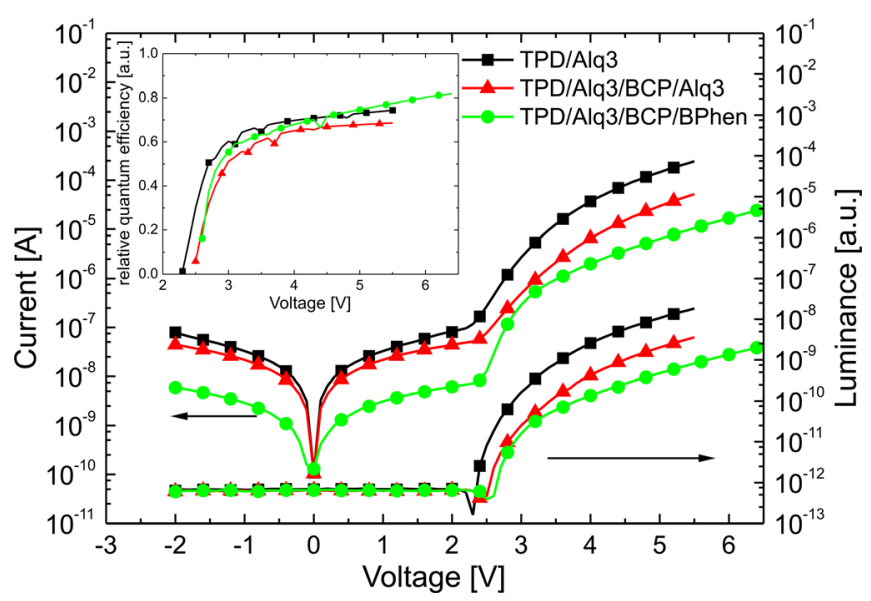

FIG. 2. (Color online) I-V-L measurements of the three different devices. The inset shows the relative quantum efficiency.

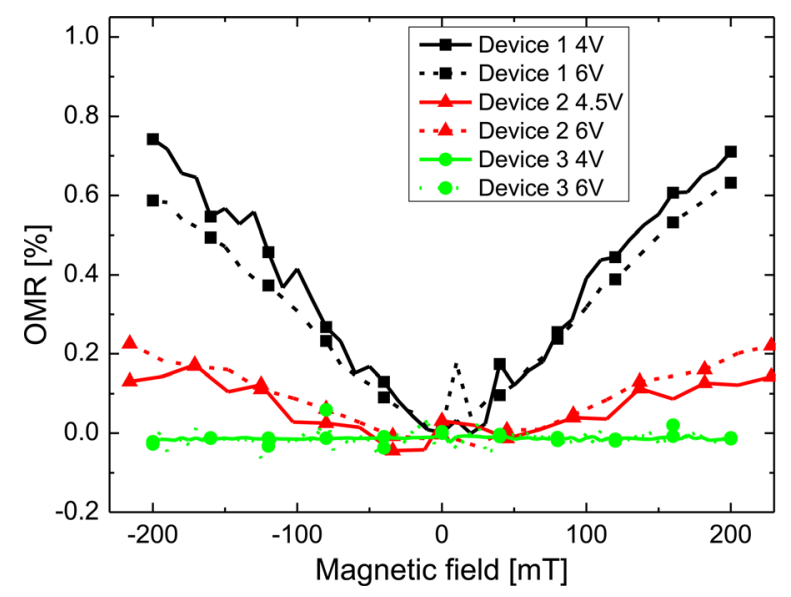

FIG. 3. (Color online) OMR measurements at different voltages. The linearity is caused by a strong high field effect.

this does not affect the following measurements. For the determination of the relative quantum efficiency, the luminance was divided by the current. Here, all three devices reveal a similar behaviour (inset in Fig. 2).

The magnetic field measurements of device 1 show a maximal OMR of $0.7 \%$ at $200 \mathrm{mT}$ and a voltage of $4 \mathrm{~V}$ (see Fig. 3). It is remarkable that the line shape is almost linear in the considered interval. Although this is in contradiction to the classical shape of the OMR, ${ }^{4}$ it can be explained with a known positive high field effect (HFE), which is sometimes superimposed on the normal OMFE. ${ }^{18,19}$ An OMR of $0.7 \%$ may seem relatively low, but this is comparable to the observations in other publications, where in pristine devices values of about $1 \%$ have been found. ${ }^{9,20}$ In Figure 4, the corresponding OML reaches up to $4.5 \%$ at $4 \mathrm{~V}$ and shows a differing behaviour. Here, the full width at half maximum is much smaller, and the influence of the HFE is less pronounced.

It should, however, be noted that after an ageing process, which is not shown here, the OMR of device 1 rose up to $4 \%$ and the HFE nearly vanished.

The maximal OMR of device 2 only amounts to $0.2 \%$ at $200 \mathrm{mT}$, but as before the graph is dominated by the HFE. The corresponding OML exhibits almost the same properties as measured on device 1 before.

Finally, device 3 shows no OMR above a value of $0.01 \%$ at the same magnetic field and voltage. It is

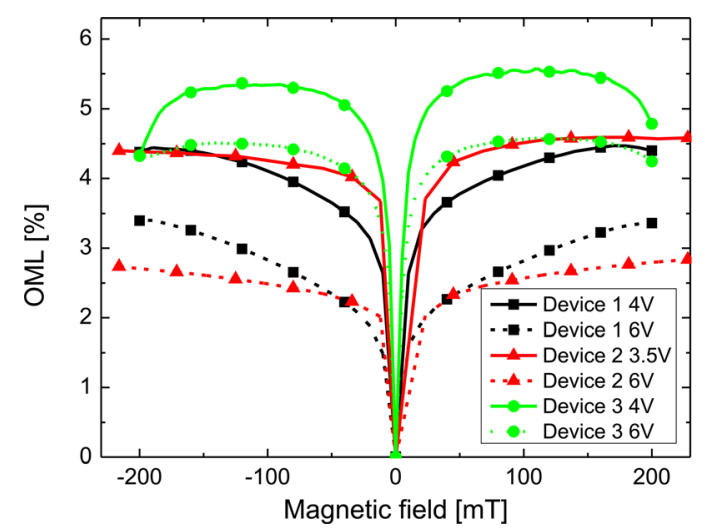

FIG. 4. (Color online) OML measurements at different voltages. 
remarkable that here the OML still has a maximal value of $5.5 \%$. It is, therefore, even more pronounced than in the other two devices.

When comparing these measurements, it can be observed that the maximal OMR of a continuous layer of $\mathrm{Alq}_{3}$ is up to $0.7 \%$. By inserting a small layer of $\mathrm{BCP}$, this value decreases to $0.2 \%$ and it vanishes when the original ETL is completely replaced by BPhen at the side of the cathode. In the case of the OMR, this behaviour can be explained by the reduction of the path, where both types of charge carriers can interact with their counterpart.

In the first device, holes and electrons can interact at a length of $70 \mathrm{~nm}$; the consequence is a relatively high OMR. Because of its blocking ability, the $2 \mathrm{~nm}$ layer of BCP in device 2 reduces the number of holes reaching the second $\mathrm{Alq}_{3}$ layer, which leads to a much smaller OMR. Nevertheless, in this case, it seems that some holes are still able to pass the BCP layer.

Finally, when replacing the $\mathrm{Alq}_{3}$ ETL by BPhen, this small number of holes is additionally blocked. Thus, the interaction between different charge carriers is limited to the $10 \mathrm{~nm}$ thin EML.

All three cases demonstrate that the reduction of the zone in which holes and electrons can interact leads to a strong reduction of the measured OMR signal. This reduction of the interaction, however, seems to have no influence on the corresponding effect on the electroluminescence. Here, all three devices show a similar OML, which has very different line shapes compared to their respective OMR. With respect to device 3 , an OML even occurs when no OMR can be measured at the same time.

All these findings lead to the conclusion that in these devices the OMR originates from phenomena in the transport layers, whereas the OML is caused by processes in the emission layer. So when both magnetic field effects are spatially separated, they can act completely independently from each other.

Previous investigations of the degradation behaviour have also given a hint that OMR and OML could have different origins. ${ }^{8}$ In consequence, we suppose that there could be an additional mechanism that is responsible for the change of the luminance.

A second finding of the present work is that an OMR can only be observed, if electrons and holes are present at the same time in a transport layer. This is in contradiction to models that try to explain the magnetic field effect on the current by the magnetic field dependent formation of bipolarons during transport. ${ }^{21}$ According to these models, holes or electrons in the separated HTL or ETL of device 3 should also form bipolarons and, therefore, cause an OMR, which is not observed. In addition, these models do not take into account the OML. Changes of the electroluminescence in a magnetic field would merely be a result of the current modification via the OMR. As has been demonstrated here, in all three devices, the OMR and OML graphs differ in almost all parameters. The magnitude is very different, and in device 3, an OML can even exist without the presence of an OMR. Furthermore, the OML shows no such distinct high field effect as the OMR, and finally, the full width at half maximum of both effects also differs.
Currently, there is no agreement on one model that can account for all aspects of OMFE in OLEDs. However, we may speculate that a combination of different models could be used to explain our observations at least qualitatively. According to an electron-hole pair model, the OML can be caused by magnetic field dependent cross sections for the formation of singlets and triplets. ${ }^{1,22}$ In a device without blocking layers, triplet excitons can then diffuse from the EML into the ETL and interact with polarons, as it is described in the triplet-polaron interaction model. ${ }^{9}$ Thus, the magnetic field dependent density of the triplet excitons has an influence on the transport of charge carriers and will cause an OMR. The introduction of an energy barrier by the blocking layer in device 3 would prevent this from happening and thus suppress the OMR without affecting the OML.

In summary, we investigated a series of OLEDs where the successive insertion of blocking layers restrains the holes and electrons from interacting with each other on their way through the organic semiconductors. It was found that this restriction of the interaction results in a decrease of the magnitude of the OMR, whereas the corresponding OML stays almost the same in all cases. This, in addition with previous findings, shows that OMR and OML can be spatially separated, and therefore, they should rather be regarded as two distinct existing effects.

Since the OMR is only measurable if holes and electrons are able to interact with each other in one layer, a model that is trying to explain the OMFE with only one type of charge carrier can a priori not account for this finding. It is, therefore, more likely that the effect is of bipolar nature.

In further studies, the method shown here could be a way to design OLEDs that allow for a separate observation of only one of the two effects,. And it could also offer the possibility to produce devices that show an enhancement of their light output, due to a magnetic field, whereas the electrical properties stay unchanged.

${ }^{1}$ J. Kalinowski, M. Cocchi, D. Virgili, P. Di Marco, and V. Fattori, Chem. Phys. Lett. 380, 710 (2003).

${ }^{2}$ T. L. Francis, Ö. Mermer, G. Veeraraghavan, and M. Wohlgenannt, New J. Phys. 6, 185 (2004).

${ }^{3}$ Ö. Mermer, G. Veeraraghavan, T. L. Francis, and M. Wohlgenannt, Solid State Commun. 134, 631 (2005).

${ }^{4}$ Ö. Mermer, G. Veeraraghavan, T. L. Francis, Y. Sheng, D. T. Nguyen, M. Wohlgenannt, A. Köhler, M. K. Al-Suti, and M. S. Khan, Phys. Rev. B 72, 205202 (2005).

${ }^{5}$ U. Niedermeier, M. Vieth, R. Pätzold, W. Sarfert, and H. von Seggern, Appl. Phys. Lett. 92, 193309 (2008).

${ }^{6}$ U. Niedermeier, Ph.D. dissertation (Technische Universität Darmstadt, 2010).

${ }^{7}$ Y. Sheng, T. D. Nguyen, G. Veeraraghavan, Ö. Mermer, M. Wohlgenannt, S. Qiu, and U. Scherf, Phys. Rev. B 74, 045213 (2006).

${ }^{8}$ T. D. Schmidt, A. Buchschuster, M. Holm, S. Nowy, J. A. Weber, and W. Brütting, Synth. Met. 161, 637 (2011).

${ }^{9}$ P. Desai, P. Shakya, T. Kreouzis, W. P. Gillin, N. A. Morley, and M. R. J. Gibbs, Phys. Rev. B 75, 094423 (2007).

${ }^{10}$ W. Brütting, S. Berleb, and A. G. Mückl, Org. Electron. 2, 1 (2001).

${ }^{11}$ T. M. Brown, J. S. Kim, R. H. Friend, F. Cacialli, R. Daik, and W. J. Feasr, Appl. Phys. Lett. 75, 1679 (1999).

${ }^{12}$ Y. Kawabe and J. Abe, Appl. Phys. Lett. 81, 493 (2002).

${ }^{13}$ Z. Zhi-lin, J. Xue-yin, Z. Wen-qing, Z. Bu-xin, and X. Shao-hong, J. Phys. D: Appl. Phys. 34, 3083 (2001).

${ }^{14}$ B. P. Rand, J. Xue, F. Yang, and S. R. Forrest, Appl. Phys. Lett. 87, 233508 (2005). 
${ }^{15}$ G. He, M. Pfeiffer, K. Leo, M. Hofmann, J. Birnstock, R. Pudzich, and J. Salbeck, Appl. Phys. Lett. 85, 3911 (2004).

${ }^{16}$ Y. Kijima, N. Asai, and S. Tamura, Jpn. J. Appl. Phys. 38, 5274 (1999).

${ }^{17}$ F. L. Bloom, W. Wagemans, M. Kemerink, and B. Koopmans, Appl. Phys. Lett. 93, 263302 (2008).

${ }^{18}$ W. Wagemans, Ph.D. dissertation (Eindhoven University of Technology, 2010).
${ }^{19}$ F. J. Wang, H. Bässler, and Z. Valy Vardeny, Phys. Rev. Lett. 101, 236805 (2008).

${ }^{20}$ U. Niedermeier, S. A. Bagnich, C. Melzer, W. Sarfert, and H. von Seggern, Synth. Met. 160, 251 (2010).

${ }^{21}$ P. A. Bobbert, T. D. Nguyen, F. W. A. van Oost, B. Koopmans, and M. Wohlgenannt, Phys. Rev. Lett. 99, 216801 (2007).

${ }^{22}$ S. A. Bagnich, U. Niedermeier, C. Melzer, W. Sarfert, and H. von Seggern, J. Appl. Phys. 106, 113702 (2009). 